\title{
The effects of nefopam with fentanyl in intravenous patient- controlled analgesia after arthroscopic orthopedic surgery: a prospective double-blind randomized trial
}

\author{
Mi Ae Jeong1, Yu Na Oh², Tae Yun Kim¹, Kyu Nam Kim¹ \\ ${ }^{1}$ Dept. of Anesthesiology and Pain Medicine, Hanyang University, Seoul, Korea \\ ${ }^{2}$ Dept. of Anesthesiology and Pain Medicine, Yonsei University, Seoul, Korea
}

BACKGROUND: Although nefopam has been used as an analgesic in Patient-controlled analgesia (PCA), combination use of nefopam and fentanyl through PCA has not been performed. Therefore, we carried out this prospective randomized, double-blind study to assess the effects of nefopam compared with ketorolac in intravenous fentanyl based patient-controlled analgesia after shoulder arthroscopic orthopedic surgery.

METHODS: 92 patients were randomly divided into two groups to receive intravenous PCA, which were nefopam group (nefopam $120 \mathrm{mg}$ and fentanyl $20 \mu \mathrm{g} / \mathrm{kg}$ in total $100 \mathrm{ml}$ ) and ketorolac group (ketorolac $2 \mathrm{mg} / \mathrm{kg}$ and fentanyl $20 \mu \mathrm{g} / \mathrm{kg}$ in total $100 \mathrm{ml}$ ). Pain assessment was assessed by a visual analog scale (VAS) and numeric rating scale (NRS). Additionally, patient satisfaction, adverse events and vital sign were observed.

RESULTS: There were no significant differences in VAS score $(\mathrm{P}=0.48)$, NRS score $(\mathrm{P}=0.15)$. Patient satisfaction did not differ between groups [8.5 (0.8) vs 8.2 (1.0), $\mathrm{P}=0.14]$. There were no significant statistical differences in incidences of nausea $(\mathrm{P}=0.72)$, vomiting $(\mathrm{P}$ $=0.46)$, urinary retention $(\mathrm{P}=0.82)$, pruritus $(\mathrm{P}=1.00)$, shivering $(\mathrm{P}=1.00)$, sweating $(\mathrm{P}=0.49)$, and dizziness $(\mathrm{P}=0.45)$ between the both groups. There were also no differences in heart rate $[78.2(7.7)$ vs $75.2(6.5), \mathrm{P}=$ 0.18 ], $\mathrm{SpO}_{2}$ [98.4 (1.8) vs 98.5 (1.9), $\mathrm{P}=0.83$ ].

Table 1. Patients demographic data

\begin{tabular}{lccc}
\hline & $\begin{array}{c}\text { Nefopam group } \\
(\mathrm{n}=46)\end{array}$ & $\begin{array}{c}\text { Ketorolac group } \\
(\mathrm{n}=46)\end{array}$ & P value \\
\hline Age & $53.3 \pm 12.8$ & $51.9 \pm 11.5$ & 0.59 \\
Sex $(\mathrm{M} / \mathrm{F})$ & $25 / 21$ & $21 / 25$ & 0.53 \\
Height $(\mathrm{cm})$ & $162.6 \pm 11.7$ & $162.8 \pm 7.9$ & 0.93 \\
Weight $(\mathrm{kg})$ & $65.3 \pm 9.7$ & $66.7 \pm 9.7$ & 0.48 \\
Aneth. time $(\mathrm{min})$ & $135.9 \pm 39.0$ & $128.5 \pm 29.3$ & 0.31 \\
Op. time (min) & $87.5 \pm 36.8$ & $80.8 \pm 26.4$ & 0.32 \\
ASA class $(1 / 2)$ & $23 / 23$ & $25 / 21$ & 0.84 \\
\hline
\end{tabular}

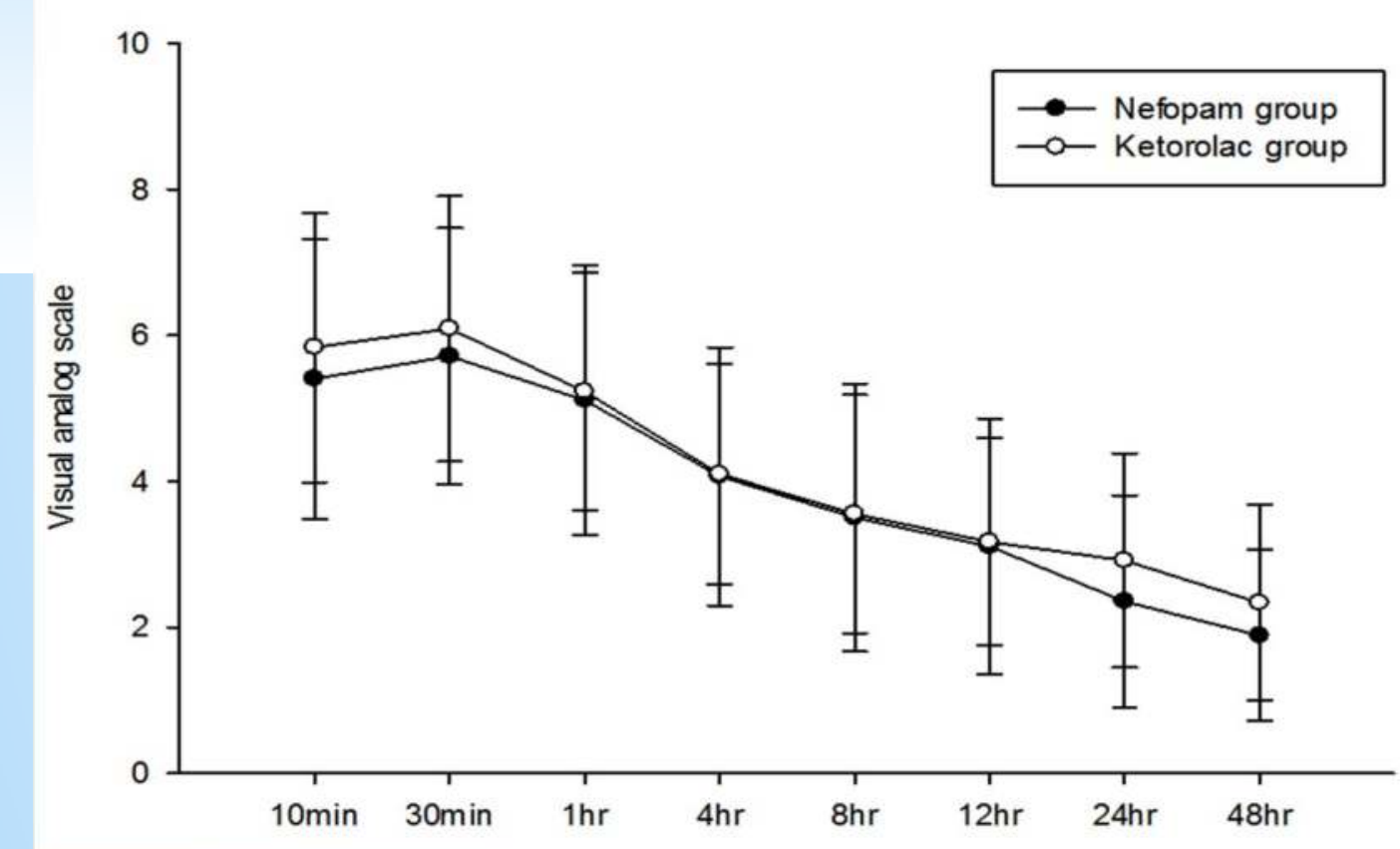

Table 2. Total PCA infusion volume ( $\mathrm{ml}$ ) and the number of pressed bolus button during $24 \mathrm{hr}$ after the operation.

\begin{tabular}{llll}
\hline & N group $(\mathrm{n}=46)$ & K group $(\mathrm{n}=46)$ & P value \\
\hline Total PCA infusion volume $(\mathrm{ml})$ & & & \\
10 minute & $0.84(0.47)$ & $1.00(0.69)$ & 0.22 \\
\hline 20 minute & $1.74(0.71)$ & $2.00(0.85)$ & 0.12 \\
1 hour & $3.28(0.99)$ & $3.58(1.16)$ & 0.18 \\
4 hour & $8.50(2.74)$ & $9.13(3.05)$ & 0.30 \\
8 hour & $13.66(3.64)$ & $14.57(4.82)$ & 0.31 \\
12 hour & $18.69(4.51)$ & $19.76(6.10)$ & 0.34 \\
24 hour & $32.84(7.17)$ & $34.44(8.02)$ & 0.32 \\
\hline The number of pressed bolus button & & & \\
10 minute & $1.50(1.97)$ & $2.26(2.51)$ & 0.11 \\
\hline 2 minute & $3.59(5.04)$ & $5.17(5.84)$ & 0.17 \\
1 hour & $6.17(8.38)$ & $8.04(7.44)$ & 0.26 \\
4 hour & $5.43(6.07)$ & $7.59(7.32)$ & 0.13 \\
\hline 8 hour & $1.71(3.15)$ & $2.88(5.47)$ & 0.21 \\
\hline 12 hour & $1.59(3.09)$ & $1.87(3.19)$ & 0.67 \\
\hline 24 hour & $3.65(9.48)$ & $3.24(4.69)$ & 0.79 \\
\hline
\end{tabular}

Table 3. Incidence of adverse events and requirement of additional treatment during 48 hours after the operation.

\begin{tabular}{llccc}
\hline & & N group $(\mathrm{n}=46)$ & K group $(\mathrm{n}=46)$ & P-value \\
\hline Adverse events & & & & \\
Nausea & PACU & $2(4)$ & $3(7)$ & 0.50 \\
& $1-4$ hours & $7(15)$ & $7(15)$ & 1.00 \\
& $4-12$ hours & $12(26)$ & $11(24)$ & 0.50 \\
& $12-24$ hours & $2(4)$ & $1(2)$ & 0.50 \\
Vomiting & $24-48$ hours & $2(4)$ & $1(2)$ & 0.50 \\
& PACU & 0 & 0 & 1.00 \\
& $1-4$ hours & $3(7)$ & $3(7)$ & 1.00 \\
& $4-12$ hours & $3(7)$ & 0 & 0.24 \\
& $12-24$ hours & 0 & 0 & 1.00 \\
Urine retention & $24-48$ hours & 0 & 0 & 1.00 \\
& PACU & $1(2)$ & 0 & 1.00 \\
& $1-4$ hours & $3(7)$ & $1(2)$ & 0.62 \\
& $4-12$ hours & $9(20)$ & $3(7)$ & 0.12 \\
Dizziness & $12-24$ hours & $6(13)$ & $2(4)$ & 0.27 \\
& $24-48$ hours & $4(9)$ & $1(2)$ & 0.36 \\
& PACU & $6(13)$ & $1(2)$ & 0.11 \\
& $1-4$ hours & $6(13)$ & $6(13)$ & 1.00 \\
& $4-12$ hours & $5(11)$ & $7(15)$ & 0.76 \\
Sweating & $12-24$ hours & $3(7)$ & $3(7)$ & 1.00 \\
Shivering & $24-48$ hours & $3(7)$ & 0 & 0.24 \\
Pruritus & & $2(4)$ & 0 & 0.49 \\
Additional treatment & & 0 & 0 & 1.00 \\
Analgesics & & 0 & 0 & 1.00 \\
Antiemetics & & $19(41)$ & $18(39)$ & 1.00 \\
Urinary catheter ins & & $7(15)$ & $6(13)$ & 1.00 \\
ertion & & $6(13)$ & $1(2)$ & 0.11 \\
\hline
\end{tabular}

CONCLUSIONS: The combinatory use of nefopam and fentanyl in PCA provided similar analgesic effects of ketorolac and fentanyl without increased adverse effects. Consequently, nefopam is considered to be an appropriate alternative drug to be co-administered with fentanyl based PCA for patients who have difficulty using Non-steroidal anti-inflammatory drugs. 